\title{
Atrial Septal Defect Presenting as Platypnea Orthodeoxia Syndrome
}

\author{
Arianne Clare Agdamag, MD ${ }^{1 *}$, Joanne Michelle Gomez, MD \\ ${ }^{1}$ Department of Internal Medicine, Rush University Medical Center, Chicago, IL 60612
}

Received Date: May 28, 2018 Accepted Date: June 12, 2018 Published Date: June 18, 2018

Citation: Arianne Clare Agdamag, Joanne Michelle Gomez (2018). Atrial Septal Defect presenting as Platypnea

Orthodeoxia Syndrome. POJ Clin Case Rep. 1(1):1-2.

*Corresponding Author: Arianne Clare Agdamag, MD, Dept. of Internal Medicine, Rush University Medical Center, 1653 W Congress Parkway, Chicago, IL, 60612, United States, E-mail: arianneclare_c_agdamag@rush.edu

\begin{abstract}
Platypnea orthodeoxia syndrome is an uncommon phenomenon characterized by dyspnea and arterial desaturation in an upright position improved with recumbency. It is due to an anatomical factor that allows for interatrial communication and a functional factor that promotes right to left shunting. Once identified, early intervention allows for rapid symptomatic improvement. This case highlights that among patients who present with positional hypoxia, POS should be considered as a possible etiology. Thorough evaluation is warranted to identify potentially reversible etiologies and guide management.

Keywords: Platypnea orthodeoxia syndrome, Atrial septal defect, Amplatzer closure device
\end{abstract}

\section{Case Report}

A 65-year-old-male with chronic lymphocytic leukemia and diffuse large $B$ cell lymphoma transformation and hepatitis $C$ complicated by stage IV liver fibrosis presented with dyspnea and abdominal pain. Physical examination showed clear breath sounds and left lower quadrant abdominal tenderness. Abdominal radiograph showed moderate stool burden while chest radiograph was unremarkable. Heparin was started empirically due to suspicion for pulmonary embolism in the setting of active malignancy and impaired mobility but was later discontinued given low probability on ventilation-perfusion (V/Q) scan. While admitted, the patient was noted to have oxygen desaturation episodes to $70 \%$ when seated upright which correlated with hypoxemia to $76 \mathrm{mmHg}$ noted on arterial blood gas. Oxygen saturation consistently improved to $100 \%$ laying supine. His hospital course was also complicated by acute stroke that presented as sudden-onset left-sided weakness. Cardiac work-up for both hypoxia and stroke consisted of transthoracic echocardiogram with bubble study which showed atrial septal defect (ASD) with massive right-to-left shunt and subsequent transesophageal echocardiogram (TEE) characterized an 11 mm ostium secundum ASD with bidirectional shunt (Figure 1). POS was attributed to large ASD for which patient underwent percutaneous closure with a $22 \mathrm{~mm}$ Amplatzer septal occluder which gradually improved his oxygenation status.

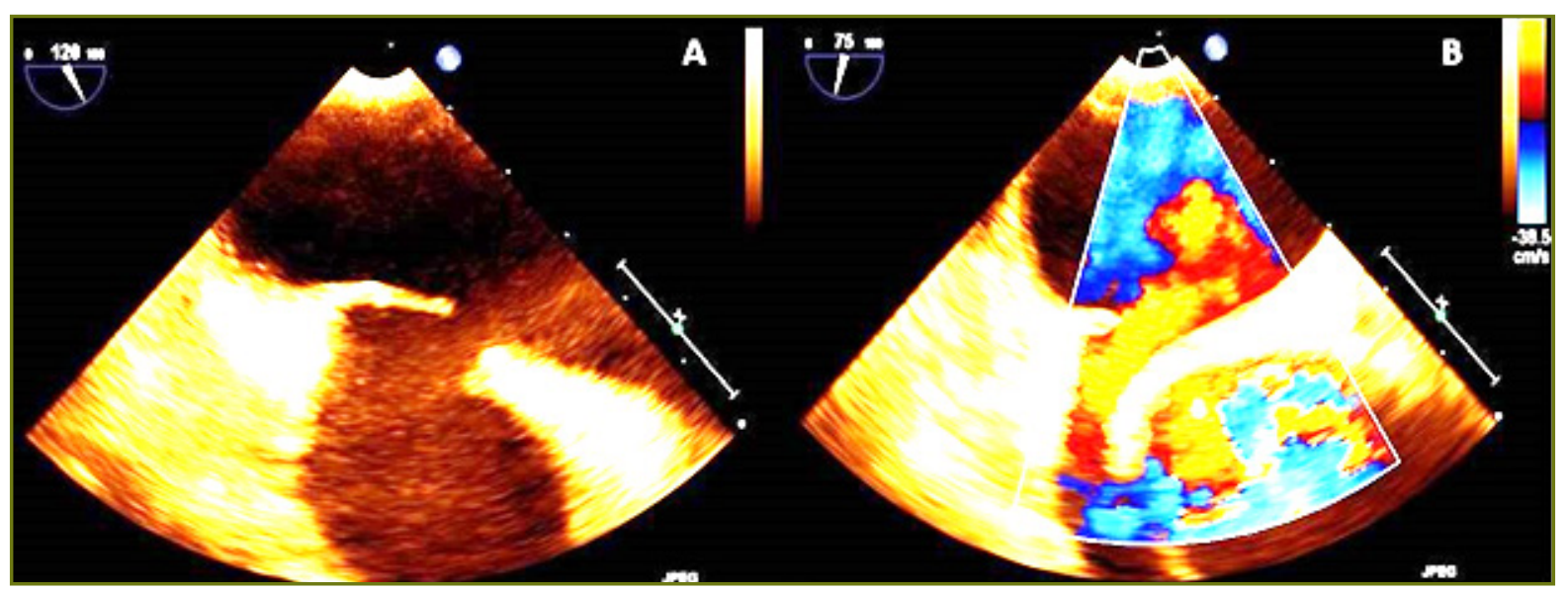

FIgURE 1: (A) Transesophageal echocardiographic image showing $11 \mathrm{~mm}$ ostium secundum atrial septal defect (B) Doppler imaging showing bi-directional flow along defect

Copyright: ${ }^{\circledR} 2018$ Arianne Clare Agdamag, et al. This is an open-access article distributed under the terms of the Creative Commons Attribution License, which permits unrestricted use, distribution, and reproduction in any medium, provided the original author and source are credited. 


\section{Discussion}

POS is a rare condition with less than 200 cases reported in literature to date [1]. Intra-cardiac shunting, pulmonary arteriovenous malformations, and ventilation/perfusion mismatch in the lungs have all been proposed underlying mechanisms of POS [2].

The pathophysiology of POS depends on the underlying etiology. Among patients with an intra-cardiac shunt, the upright position leads to a marked displacement of the interatrial septum and atrial septal defect causing preferential flow through the septal deformation magnifying the amount of deoxygenated blood that goes to the left sided circulation [3].

Among cardiaccauses, patent foramen ovale is the most frequent lesion and ASD ranks second [4]. TEE, the preferred diagnostic study to visualize cardiac defects or aneurysms, confirmed an ostium secundum ASD in this patient. Management, as in this case, often involves percutaneous closure. In this patient with chronic liver disease, hepatopulmonary syndrome leading to pulmonary AVM may be a consideration if no improvement was observed after ASD closure. With a 4.6\% POS recurrence risk in 3 years post-shunt correction, prognosis remains good for those who have POS secondary to correctable defects such as ASD [5].

The case highlights the significance of recognition of this rare syndrome that is readily observed at bedside. Appropriate diagnostic work-up to identify the causes that may dramatically improve symptoms with timely intervention.

Declaration: All authors have nothing to disclose.

\section{References:}

1. Akin E, Krüger P, Braun E, et al. The Platypnea-Orthodeoxia Syndrome. Eur Rev Med Pharmacol Sci. 2014;18(18):2599604.

2. Ali OM, Agarwal A, Akram S. Platypnea Orthodeoxia: a 'laidback' Case of Dyspnoea. BMJ Case Rep. 2013. Published online: January 28, 2013 doi:10.1136/bcr-2012- 007810.

3. Henkin S, Negrotto S, Pollak PM, et al. Platypnea-Orthodeoxia Syndrome: Diagnostic Challenge and the Importance of Heightened Clinical Suspicion. Tex Heart Inst J. 2015;42(5):498-501. doi:10.14503/THIJ-14-4596

4. Kazawa S, Enomoto T, Suzuki N, et al. Platypnea-orthodeoxia Syndrome in a Patient with an Atrial Septal Defect: The Diagnosis and Choice of Treatment. Intern Med. 2017; 56(2):169-173. doi:10.2169/internalmedicine.56.7728

5. Knapper JT, Schultz J, Das G, et al. Cardiac platypnea-orthodeoxia syndrome: an often unrecognized malady. Clin Cardiol. 2014;37(10):645-9. doi 10.1002/clc.22301 